Chirurg 2016 $87: 1078$

DOI 10.1007/s00104-016-0321-z

Online publiziert: 4. November 2016

(c) Springer-Verlag Berlin Heidelberg 2016

CrossMark
M. Schrempf $\cdot$ M. Anthuber

Klinik für Allgemein-, Viszeral- Transplantationschirurgie, Klinikum Augsburg, Augsburg, Deutschland

\section{Prophylaktischer Doppelbypass beim nichtresektablen Pankreaskarzinom}

\section{Ein Gewinn für den Patienten?}

\section{Originalpublikation}

Insulander J, Sanjeevi S, Haghighi M et al (2016) Prognosis following surgical bypass compared with laparotomy alone in unresectable pancreatic adenocarcinoma. $\mathrm{Br} \mathrm{J}$ Surg 103:1200-1208

Hintergrund. Bis zu $33 \%$ aller präoperativ als resektabel gewerteten Pankreaskarzinome werden intraoperativ aufgrund von Metastasen oder lokal fortgeschrittenem Wachstums als inoperabel eingestuft. In diesen Fällen stellt sich die Frage, ob ein prophylaktischer Doppelbypass (PDB) bestehend aus Hepatikojejunostomie und Gastroenterostomie angelegt oder die Operation als explorative Laparotomie (EL) beendet werden sollte.

Methoden. In dieser retrospektiven Studie wurden Daten von Pankreaskarzinompatienten ausgewertet, die zwischen 2004 und 2013 eine operative Therapie erhielten. Nur histologisch gesicherte duktale Adenokarzinome des Pankreas (PDAC) wurden eingeschlossen. Die Entscheidung zur Anlage eines PDB oder Beendigung der Operation als EL blieb dem Operateur überlassen. Eine Chemotherapie wurde für alle inoperablen Patienten empfohlen, deren klinischer Zustand eine Chemotherapie zuließ.

Ergebnisse. Von 503 primär als resektabel bewerteten PDAC wurden 104 Karzinome intraoperativ als nichtresektabel eingestuft (Resektionsrate 79,3 \%). $74 \mathrm{~Pa}$ tienten erhielten einen PDB und 30 eine
EL. Die 30-Tage-Mortalität und das Gesamtüberleben waren für PDB und EL vergleichbar. $49 \%$ der Patienten erhielten eine palliative Chemotherapie. Der Anteil an Patienten, die eine palliative Chemotherapie erhielten, ebenso wie das Intervall bis zum Beginn der Chemotherapie unterschieden sich nicht zwischen den beiden Gruppen. In der PDB-Gruppe zeigten sich eine signifikant längere Operationsdauer, ein höherer Blutverlust sowie eine längere Krankenhausverweildauer. Patienten, die eine postoperative Chemotherapie erhielten, wiesen ein signifikant längeres Gesamtüberleben als Patienten ohne Chemotherapie auf (11,6 vs. 3,0 Monate; $p<0,001)$. Patienten mit Chemotherapie nach EL zeigten im Vergleich zu Patienten mit Chemotherapie nach PDB ein signifikant längeres medianes Gesamtüberleben (16,3 vs. 10,3 Monate, $p=0,040)$.

Diskussion und Fazit. In der vorliegenden Studie hatte die Anlage eines PDB beim nichtresektablen PDAC einen negativen Effekt auf das Gesamtüberleben von Patienten, die eine palliative Chemotherapie erhielten. Diese Ergebnisse werden durch Daten von Spanheimer et al. gestützt, die für palliative Eingriffe beim nichtresektablen PDAC ein reduziertes Gesamtüberleben nachwiesen [1]. In einer weiteren retrospektiven Arbeit war die Anlage eines PDB im Vergleich zu einer Wait-and-see-Strategie und endoskopischer Intervention im Falle von Obstruktionen mit einer höheren Morbidität assoziiert [2]. Auch wenn aus prognostischer Sicht die Anlage eines PDB im
Vergleich zur EL ungünstiger erscheint, ermöglicht es die aktuelle, überwiegend retrospektive Datenlage nicht, palliative operative Maßnahmen beim nichtresektablen PDAC grundsätzlich abzulehnen. Vielmehr müssen jedem Patienten präoperativ die therapeutischen Optionen im Falle eines inoperablen Tumors aufgezeigt werden und intraoperativ eine Beurteilung der individuellen Situation erfolgen.

\section{Korrespondenzadresse}

Prof. Dr. M. Anthuber

Klinik für Allgemein-, Viszeral- Transplantationschirurgie, Klinikum Augsburg

Stenglinstr. 2, 86156 Augsburg, Deutschland matthias.anthuber@klinikum-augsburg.de

Interessenkonflikt. M. Schrempf und M. Anthuber geben an, dass kein Interessenkonflikt besteht.

\section{Literatur}

1. Spanheimer PM, Cyr AR, Liao J et al (2014) Complications and survival associated with operative procedures in patients with unresectable pancreatic head adenocarcinoma. J Surg Oncol 109:697-701

2. Williamsson C, Wennerblom J, Tingstedt B, Jönsson $C$ (2016) A wait-and-see strategy with subsequent self-expanding metal stent on demand is superior to prophylactic bypass surgery for unresectable periampullary cancer. HPB (Oxford) 18(1):07-112 